\title{
An Integration of Train Timetabling, Platforming and Routing-Based Cooperative Adjustment Methodology for Dealing with Train Delay
}

\author{
Yinggui Zhang*, Zengru Chen*, Min An ${ }^{\dagger+}$ \\ and Aliyu Mani Umar* \\ *School of Traffic and Transportation Engineering \\ Central South University \\ Changsha 410004, P. R. China \\ ${ }^{\dagger}$ School of Science, Engineering and Environment \\ University of Salford \\ Manchester M5 $4 W T, U K$ \\ +m.an@salford.ac.uk
}

Received 15 February 2020

Revised 2 March 2020

Accepted 22 March 2020

\begin{abstract}
Train delay is a serious issue that can spread rapidly in the railway network leading to further delay of other trains and detention of passengers in stations. However, the current practice in the event of the trail delay usually depends on train dispatcher's experience, which cannot manage train operation effectively and may have safety risks. The application of intelligent railway monitor and control system can improve train operation management while increasing railway safety. This paper presents a methodology in which train timetabling, platforming and routing models are combined by studying the real-time adjustment and optimization of highspeed railway in the case of the train delay in order to produce a cooperative adjustment algorithm so that the train operation adjustment plan can be obtained. MATLAB computer programs have been developed based on the proposed methodology and adjustment criteria have been established from knowledge data bases in order to calculate optimized solutions. A case study is used to demonstrate the proposed methodology. The results show that the proposed method can quickly adjust the train operation plan in the case of the train delay, restore the normal train operation order, and reduce the impact of train delay on railway network effectively and efficiently.
\end{abstract}

Keywords: Train delay; railway timetabling; train platforming; train routing; optimization models and algorithms.

\section{Notations}

$i:$ Trains, $i \in \boldsymbol{I}, \boldsymbol{I}=\{1,2, \ldots, k\}$;

$s:$ Stations, $s \in \boldsymbol{S}, \boldsymbol{S}=\{1,2, \ldots, m\}$; 
AQ: Please check edit.

\section{Y. Zhang et al.}

$k$ : Total train number;

$m$ : Total station number;

$u_{s}$ : Platform track in station $s, u_{s} \in \boldsymbol{U}_{s}, \boldsymbol{U}_{s}=\left\{1_{s}, 2_{s}, \ldots, n_{s}\right\}$;

$n_{s}$ : Total number of platform tracks at station $s$;

$r_{s}$ : Train routes at station $s, r_{s} \in \boldsymbol{R}_{s}, \boldsymbol{R}_{s}=\left\{1_{s}, 2_{s}, \ldots, w_{s}\right\}$;

$w_{s}$ : Total number of train routes at station $s$;

$t_{i s}^{a}$ : Original scheduled time for train $i$ to arrive at station $s$;

$t_{i s}^{d}$ : Original scheduled time for train $i$ to depart from station $s$;

$x_{i u}^{s}= \begin{cases}1, & \text { if train } i \text { occupies platform track } u \text { at station } s \text { in the original plan } \\ 0, & \text { otherwise }\end{cases}$

$X_{i u}^{s}$ : The platform track number occupied by train $i$ at station $s$ in the original plan, when $x_{i u}^{s}=1, X_{i u}^{s}=u$;

$o_{i r}^{s}= \begin{cases}1, & \text { if train } i \text { occupies route } r \text { at station } s \text { in the original plan } \\ 0, & \text { otherwise }\end{cases}$

$h_{u}^{s}$ : The route to the platform track $u$ in stations, $h_{u}^{s} \in \boldsymbol{H}_{u}^{s}, \boldsymbol{H}_{u}^{s}=\left\{1,2, \ldots, g_{u}\right\}$;

$g_{u}$ : Total number of routes to platform track $u$ at station $s$;

$t_{s, s+1}^{i}$ : Running time of train $i$ between station $s$ and station $s+1$;

$\tau_{i s}$ : Minimum stop time of train $i$ at station $s$;

$\tau_{s}^{z}:$ Tracking interval of station $s$;

$\tau_{s}^{d}:$ Arriving interval of station $s$

$\tau_{u s}$ : The interval between two trains using track $u$ in station $s$;

$t_{i s}^{\prime a}:$ Adjusted time for train $i$ to arrive at station $s$;

$t_{i s}^{\prime d}:$ Adjusted time for train $i$ to depart from station $s$;

$\boldsymbol{V} \boldsymbol{L}_{i}^{s}$ : Train numbers at station $s$ during $t_{i s}^{\prime d}-t_{i s}^{\prime a}$ period;

$x_{i u}^{\prime s}= \begin{cases}1, & \text { if train } i \text { occupies platform track } u \text { at station } s \text { in the adjusted plan } \\ 0, & \text { otherwise }\end{cases}$

$X_{i u}^{\prime s}$ : The platform track number occupied by train $i$ at station $s$ in the adjusted plan, when $x_{i u}^{\prime s}=1, X_{i u}^{\prime s}=1$;

$o^{s}= \begin{cases}1, & \text { if train } i \text { occupies route } r \text { at station } s \text { in the adjusted plan } ; \\ 0, & \text { otherwise }\end{cases}$

$y_{i u h}^{\prime s}= \begin{cases}1, & \text { if train } i \text { passes through route } h \text { to track } u \text { in the adjusted plan } \\ 0, & \text { otherwise }\end{cases}$

$y_{i}= \begin{cases}1, & \text { if train } i \text { is delayed } \\ 0, & \text { otherwise }\end{cases}$

$\theta_{i}= \begin{cases}1, & \text { if }\left(t_{i s}^{\prime d}-t_{i s}^{d}\right)<T, T \text { is a constaint } \\ 0, & \text { otherwise }\end{cases}$

where $t_{i s}^{\prime a}, t_{i s}^{\prime d}, x_{i u}^{\prime s}, o_{i r}^{\prime s}, y_{\text {iuhs }}^{\prime} y_{i}, \theta_{i}$ are the decision variables. 


\section{Introduction}

Trains in a railway network must be operated by following systematic predetermined schedules based on railway network capacity. If a train is delayed, it would affect other trains' arrivals and departures at stations. However, the change of timetabling because of such a delay will also affect the arrangement of platforms and route plans in the railway network [1].

Studies have been conducted on the optimization of train timetabling in order to manage and control the delay of trains. Corman et al. [2] and Luan et al. [3] investigated the problems of railway transportation interference and train delay management and proposed a method of integration of train scheduling and delay for real-time railway traffic control. Cacchiani et al. [4] studied the recovery of real-time interference and management of railways and developed a recovery model for realtime railway rescheduling by taking train scheduling and train delay into consideration. Yang et al. [5] developed a method for minimizing the total delay time at the departure station and the residence time of intermediate stations as an objective function and established a collaborative optimization model for the integration of train stop planning in order to solve train dispatching problems. However, these studies only considered effects of the train delay by taking train scheduling into consideration, but train platforming and routing plans are not considered.

Some of researches have been done which considered train platforming and routing and problems in dealing with the train delay. Carey et al. $[6,7]$ studied train operation timetable and arrival/departure track arrangements in large hub stations and developed a model for train scheduling for busy complex stations in railway network. Zhang et al. [8] combined train routing, interlocking and train platform compilation rules, and further developed a real-time adjustable and controllable collaborative optimization model for train platforming in railway stations. Samà et al. [9] proposed an integer linear programming model for real-time adjustment and re-arrangement of train platforms and train routes to solve the problem because of the train delay.

However, the current studies address either the optimization of train timetable and the adjustment of train delay or the optimization of train platforming and routes and the adjustment of train delay, but the adjustment of train scheduling, platforming and routing plans are not considered together, which is important in train operation management. This paper presents the recent development of a cooperative adjustment method in the case of the train delay in which train timetabling, platforming and routing plans are taken together with railway network capacity into consideration in order to restore normal train operation order and reduce the impact of train delay effectively. MATLAB computer programs have been developed based on the proposed methodology and adjustment criteria have been established from knowledge data bases in order to calculate optimized solutions. A case example is used to demonstrate the proposed methodology. 


\section{Modeling Development of Train Timetabling, Platforming and Routing}

The train optimization timetabling, platforming and routing models are described in this section, respectively, which will be used to establish a cooperative adjustment model. It should be noted that the parameters are considered in the proposed model which take all influential factors from the recorded databases into account.

\subsection{Train timetabling optimization model}

\subsubsection{Objective functions}

When the train delay occurs, the goal of adjustment is to minimize the total train delay time. Törnquist and Persson [10] discussed the propagation mode of interference and took corresponding measures to minimize the number of affected trains. Therefore, both the number of delayed trains and the total train delay time in the process of train delay adjustment need to be considered. The number of delayed trains can be calculated to minimize train delay time

$$
\operatorname{Min}: \mathrm{Z}_{1}^{1}=\sum_{i=1}^{k} y_{i}
$$

The total train delay time can be obtained by

$$
\operatorname{Min}: \mathrm{Z}_{1}^{2}=\sum_{i=1}^{k} \sum_{s=1}^{m}\left[\left(t_{i s}^{\prime a}-t_{i s}^{a}\right)+\left(t_{i s}^{\prime d}-t_{i s}^{d}\right)\right] .
$$

In the process of adjustment decision, the priority is to reduce the number of delayed trains in order to ensure train operation recovery quickly. Assume $\alpha$ is a weight factor, its value should be large enough to ensure the priority of the total number of delayed trains can be minimized, i.e. reducing the number of delayed trains. Variable $\theta_{i}$, value is between 0 and 1 is used to denote that if the delay time of a train is longer

AQ: Can we change Z

variable to italics in equations 1,2 , 3,10 to 14,22 to $24,26 ?$ [Global]. than $T$, the number of delayed trains will not be considered, which aims to minimize total delay time of the trains instead. Therefore, the objective function of the timetabling model can be established

$$
\text { Min : } \mathrm{Z}_{1}=\sum_{i=1}^{k}\left\{\alpha \theta_{i} y_{i}+\sum_{s=1}^{m}\left[\left(t_{i s}^{\prime a}-t_{i s}^{a}\right)+\left(t_{i s}^{\prime d}-t_{i s}^{d}\right)\right]\right\} \text {. }
$$

\subsubsection{Restraint conditions}

Suppose two trains $i$ and $j$ arrive in station $s$ successively, the timetabling model needs to satisfy the following constraints according to the rules of train diagram formulation [2]. 
(1) In any station, the stop time of a train at the station needs to meet the minimum stopover time requirement specified by the train

$$
t_{i s}^{\prime d}-t_{i s}^{\prime a} \geq \tau_{i s} \quad \forall s \in \boldsymbol{S},
$$

where $t_{i s}^{\prime a}$ is the adjusted time for train $i$ to arrive at station $s, t_{i s}^{\prime d}$ is the adjusted time for train $i$ to depart from station $s, \tau_{i s}$ is the stop time of train $i$ at station $s$, and $\boldsymbol{S}$ denotes all of stations in the railway network.

(2) In any station, the shortest interval between sending two adjacent co-directional trains in the station should meet the requirement of train tracking interval

$$
t_{i s}^{\prime d}-t_{j s}^{\prime d} \geq \tau_{s}^{z} \quad \forall s \in \boldsymbol{S}
$$

where $t_{i s}^{\prime d}$ is the adjusted time for train $i$ to arrive at station $s, t_{j s}^{\prime d}$ is the adjusted time for train $j$ to depart from station $s, \tau_{s}^{z}$ is the track interval at station $s$, and $\boldsymbol{S}$ denotes all of stations in the railway network.

(3) In any station, the continuous arrival of two adjacent co-directional trains at the station should satisfy arriving interval

$$
t_{i s}^{\prime a}-t_{j s}^{\prime a} \geq \tau_{s}^{d} \quad \forall s \in \boldsymbol{S}
$$

where $t_{i s}^{\prime a}$ is adjusted time for train $i$ to arrive at station $s, t_{j s}^{\prime a}$ is adjusted time for train $j$ to arrive at station $s, \tau_{s}^{d}$ is arrival interval at station $s$, and $\boldsymbol{S}$ denotes all of stations in the railway network.

(4) In any station, the interval between the continuous occupancy of the platform track $u$ should satisfy

$$
t_{i s}^{\prime d}-t_{j s}^{\prime a} \geq \tau_{u s} \quad \forall s \in S,
$$

where $t_{i s}^{\prime d}$ is adjusted time for train $i$ to depart from station $s, t_{j s}^{\prime a}$ is adjusted time for train $j$ to arrive at station $s, \tau_{u s}$ is the interval between the continuous occupancy of the platform track $u$ at station $s$, and $\boldsymbol{S}$ denotes all of stations in the railway network.

(5) In any station, the adjusted departure time of the train shall not be earlier than the original planned departure time

$$
t_{i s}^{d} \leq t_{i s}^{\prime d} \quad \forall s \in S
$$

where $t_{i s}^{\prime d}$ is the adjusted time for train $i$ to depart from station $s, t_{i s}^{d}$ is original scheduled time for train $i$ to depart from station $s$, and $\boldsymbol{S}$ denotes all of stations in the railway network.

(6) The arrival time of the train at station $s+1$ is its departure time from station $s$ plus its running time in the section between stations $s$ and $s+1$, which can be calculated by

$$
t_{i(s+1)}^{\prime a}-t_{i s}^{\prime d}=t_{s, s+1}^{i} \quad \forall s \in S \text { and } s<m,
$$

where $t_{i(s+1)}^{\prime a}$ is the adjusted time for train $i$ to arrive at station $s+1, t_{i s}^{\prime d}$ is the adjusted time for train $i$ to depart from station $s, \boldsymbol{S}$ denotes all of stations in the 
railway network, and $m$ is the total number of stations that the train $i$ stops in the railway network.

The timetabling optimization model can be established according to the objective function Eq. (3) and constraints Eqs. (4) to (9) as

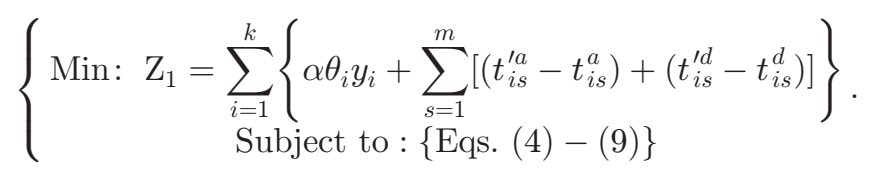

\subsection{Platforming and routing optimization models}

\subsubsection{Objective function of platforming model}

When train delays occur, the frequent train timetable changes cause stations to fail receiving and dispatching trains in accordance with the original platform plan. Therefore, the platform plan needs to be adjusted based on the changes of timetable sue to the train delay. The volatility and equalization of platform plan are currently used to measure the effect of the adjusted platform plan.

The volatility of the platform plan reflects the difference between the adjusted platform plan and the original one. The greater the value of volatility of the platform plan is, the greater the difference is between the adjusted platform plan and the original one. it is desirable to obtain a less volatile adjustment plan in order to reduce the adjustment workload because of the train delay and quickly restore train operation in the stations. Suppose the platform track occupied by train $i$ at station $s$ in the original platform plan be $X_{i u}^{s}$ and the platform track occupied by train $i$ at station $s$ in the adjusted platform plan be $X_{i u}^{\prime s}$, the volatility of the platform plan can be described as

$$
\text { Min: } \mathrm{Z}_{2}^{1}=\frac{1}{k} \sum_{i=1}^{k}\left(X_{i u}^{\prime s}-X_{i u}^{s}\right)^{2} \text {. }
$$

The platform tracks are usually numbered in a particular order. Therefore, the smaller value of $\left|X_{i u}^{\prime s}-X_{i u}^{s}\right|$ is, the closer to the original platform plan is. In other words, the platform track allocated to train $i$ at station $s$ in the adjusted platform plan is closer to the original platform plan.

The equalization of the platform plan reflects the degree of difference in the number of occupations of each platform track in a station during a certain period. It can be described as how many times that platform track is used in a certain period [11], which can be expressed as

$$
\text { Min : } \mathrm{Z}_{2}^{2}=\frac{1}{n_{s}} \sum_{u=1}^{n_{s}}\left(\sum_{i=1}^{k} x_{i u}^{s}-\frac{k}{n_{s}}\right)^{2} .
$$


Taking the volatility of the platform plan and the equalization of the platform plan into consideration, and by using the method of linear weighted summation, the two objectives Eqs. (11) and (12) are transformed into a single objective. Suppose the weight factors $\beta_{1}$ and $\beta_{2}\left(\beta_{1}+\beta_{2}=1, \beta_{1}>>\beta_{2}\right)$ for each objective [12], the objective function of the platforming model is

$$
\operatorname{Min}: \mathrm{Z}_{2}=\beta_{1} \frac{1}{k} \sum_{i=1}^{k}\left(X_{i u}^{\prime s}-X_{i u}^{s}\right)^{2}+\beta_{2} \frac{1}{n_{s}} \sum_{u=1}^{n_{s}}\left(\sum_{i=1}^{k} x_{i u}^{s}-\frac{k}{n_{s}}\right)^{2} .
$$

\subsubsection{Objective function of routing model}

A route plan refers to determining a route for train $i$ to arrive, depart or pass through station $s$, such a route is called as a train route. When train $i$ arrives or leaves the station $s$, a train route needs to be used to guide the train into or out of its corresponding platform track. Therefore, if the platform plan changes, the route plan needs to be adjusted accordingly. The higher value of equalization of the route plan is, the stronger its anti-interference ability is [13]. Therefore, similar to the equalization of the platform plan as described in Sec. 2.2.1, in order to reduce the possibility of secondary interference in the adjusted route plan, the effect of the adjusted route plan is measured by the equalization.

When the platform track $u$ occupied by train $i$ in station $s$ is determined, all the train entry routes connected with the platform track $u$ can be obtained, and then a adjusted route $y_{\text {uih }}^{\prime s}$ with the highest equalization value of the adjusted route plan can be selected. The objective function of the routing model is

$$
\operatorname{Min}: \mathrm{Z}_{3}=\sum_{u=1}^{n_{s}} \sum_{h=1}^{g_{u}}\left(\sum_{i=1}^{k} y_{i u h}^{\prime s}-\frac{\sum_{i=1}^{k} x_{i u}^{\prime s}}{g_{u}}\right)^{2}
$$

\subsubsection{Restraint conditions}

The platforming and routing models need to meet station operation requirements and satisfy the following constraints:

(1) Any station must ensure that all trains have a platform track and a route for receiving and departing

$$
\begin{gathered}
\sum_{u=1}^{n_{s}} \sum_{i=1}^{k} x_{i u}^{\prime s}=k \quad \forall s \in \boldsymbol{S}, \\
\sum_{r=1}^{r_{s}} \sum_{i=1}^{k} o_{i r}^{\prime s} \sum_{h=1}^{g_{u}} y_{i u h}^{\prime s}=k \quad \forall s \in \mathbf{S},
\end{gathered}
$$

where $x_{i u}^{\prime s}$ is the platform track allocated for train $i$ at station $s$ in the adjusted platform plan, $k$ is total number of trains, $g_{u}$ is the total number of routes to platform track $u$ at station $s$, and $n_{s}$ is total number of platform tracks at station $s$. 
$Y$. Zhang et al.

(2) In any station, each train must have one and only one track for its reception and departure

$$
\sum_{u=1}^{n_{s}} x_{i u}^{\prime s}=1 \quad \forall s \in \boldsymbol{S}
$$

(3) In any station, a platform track can only be occupied by one train in a fixed period, that is, the train in assembly $\boldsymbol{V} \boldsymbol{L}_{i}^{s}$ cannot occupy the same platform track

$$
\sum_{i \in \boldsymbol{V} \boldsymbol{L}_{i}^{s}} x_{i u}^{\prime s} \leq 1 \quad \forall s \in \boldsymbol{S}
$$

where $\boldsymbol{V} \boldsymbol{L}_{i}^{s}$ is train assemblage at station $s$ during $t_{i s}^{\prime d}-t_{i s}^{\prime a}$ period.

(4) In any station, each train must be one and only one route for its reception and departure

$$
\sum_{r_{s}=1}^{w_{s}} o_{i r}^{\prime s}=1 \quad \forall s \in \boldsymbol{S}
$$

where $r_{s}$ is train routes at station $s, r_{s} \in \boldsymbol{R}_{s}, \boldsymbol{R}_{s}=\left\{1_{s}, 2_{s}, \ldots, w_{s}\right\}$, and $w_{s}$ is the total number of train routes at station $s$.

(5) In any station, a route can only be occupied by one train in a fixed period, that is, the train in assembly $\boldsymbol{V} \boldsymbol{L}_{i}^{s}$ cannot occupy the same route

$$
\sum_{i \in \boldsymbol{V} \boldsymbol{L}_{i}^{s}} o_{i r}^{\prime s} \leq 1 \quad \forall s \in \boldsymbol{S} .
$$

(6) Suppose two trains $i$ and $j$ arrive station $s$ successively, conflict routes cannot be opened in any station at the same time

$$
\sum_{i \in \boldsymbol{V} \boldsymbol{L}_{i}^{s}} o_{i r}^{\prime s} \cap \sum_{j \in \boldsymbol{V} \boldsymbol{L}_{i}^{s}} o_{j r}^{\prime s}=\phi, \quad j=i+1 \quad \forall s \in \boldsymbol{S} .
$$

Based on the above objective functions and constraints, the platforming and routing models can be established as

$$
\text { Platforming }\left\{\begin{array}{l}
\text { Min: } Z_{2}=\beta_{1} \frac{1}{k} \sum_{i=1}^{k}\left(X_{i u}^{\prime s}-X_{i u}^{s}\right)^{2}+\beta_{2} \frac{1}{n_{2}} \sum_{u=1}^{n_{s}}\left(\sum_{i=1}^{k} x_{i u}^{s}-\frac{k}{n_{s}}\right)^{2}, \\
\text { Subject to: Eqs. }(15)-(21)
\end{array}\right.
$$

$$
\text { Routing }\left\{\begin{array}{l}
\text { Min: } Z_{3}=\sum_{u=1}^{n_{s}} \sum_{h=1}^{g_{u}}\left(\sum_{i=1}^{k} y_{i u h}^{\prime s}-\frac{\sum_{i=1}^{k} x_{i u}^{\prime s}}{g_{u}}\right)^{2} . \\
\text { Subject to: Eqs. }(15)-(21)
\end{array}\right.
$$




\section{The Proposed Cooperative Adjustment Model}

The occurrence of perturbation in a railway network that requires the adjustment of train timetable usually affects the platforming and routing plans. In other words, adjustments of train timetabling, platforming and routing plans in the case of the train delay requires adjusting the arrival and departure time of all trains to ensure a conflict-free operation. Therefore, it is necessary to combine timetabling, platforming and routing models together to establish a cooperative adjustment model.

Let $t_{i s}^{\prime a}$ and $t_{i s}^{\prime d}$ be coupling factors, the cooperative adjustment model can be established by combing Eqs. (10), (22) and (23) as

$$
\text { Cooperative Adjustment Model }\left\{\begin{array}{l}
\text { Timetabling Min: } \mathrm{Z}_{1}\left(t_{i s}^{\prime a}, t_{i s}^{\prime d}\right) \\
\text { Platfroming Min: } \mathrm{Z}_{2}\left(t_{i s}^{\prime a}, t_{i s}^{\prime d}, x_{i u}^{\prime s}\right) \\
\text { Routing Min: } \mathrm{Z}_{3}\left(t_{i s}^{\prime a}, t_{i s}^{\prime d}, x_{i u}^{\prime s}, y_{i u h}^{\prime s}\right) \\
\text { Subject to: }\{\text { Eqs. }(4)-(9), \text { and }(15)-(21)\}
\end{array} .\right.
$$

The developed cooperative adjustment model consists of three sub-models, i.e. Train timetabling, platforming and routing models as described in Sec. 2. In other words, the train arrival and departure times, platform and route schemes at a station interact and restrict each other. The solution algorithm of each sub-model needs to be developed and then the final algorithm of cooperative adjustment model can be designed to realize the real-time and fast adjustment of trains.

\subsection{Train timetabling algorithm}

As described in Sec. 2.2.1, the timetabling model is established to minimize the number of the delayed trains, i.e. Eq. (1), and the total delay time, i.e. Eq. (2). These two objectives can be achieved by

- calculating the earliest possible departure time of the delayed train $i$ according to its arrival time $t_{i s}^{\prime a}$ and its minimum stopping time $\tau_{i s}$ at station $s$. Figure 1 shows relationship between the delayed train and its subsequent trains where $a, b, c, d$ and $e$ represent the subsequent trains sorted according to the original train schedule.

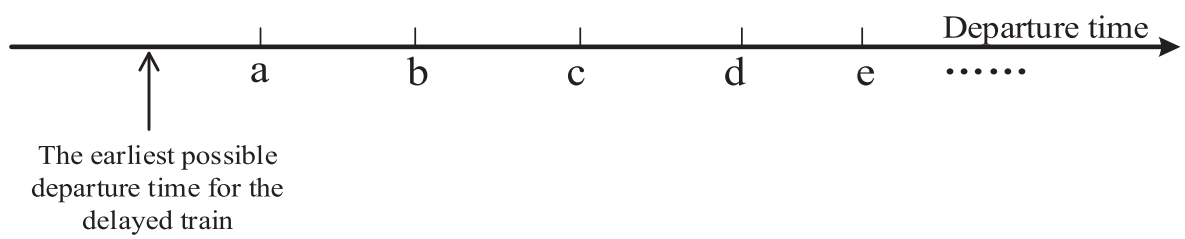

Fig. 1. Relationship between the delayed train and its subsequent trains. 
- comparing the departure times of the adjacent $j$ trains, if there is a feasible departure interval time existing, the interval time will be chosen as the departure interval of the delayed train. For example, if $j=2$, assuming that the departure time interval of trains $b$ and $c$ is large enough to satisfy the time interval requirements after inserting the delayed train $i$ into the interval, then the interval $(b, c)$ between trains $b$ and $c$ is considered as a feasible departure interval of the delayed trains as shown in Fig. 2.

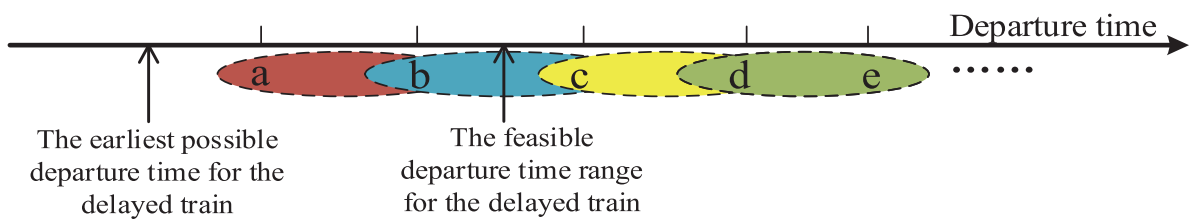

Fig. 2. Search strategy, when $j=2$.

However, if there is no feasible interval after searching all intervals when $j=2$, then $j=j+1$ and re-search until finding a solution. Therefore, when a $j+1$ adjacent train has a feasible interval, after inserting the delayed train into this interval, departure times of all trains need to move backward except the first and last train. However, all trains are not allowed to depart in advance. For example, assuming that trains $c, d$ and $e$ constitute a feasible interval when $j=j+1=3$, after the delayed train is inserted into this interval, the departure time of the subsequent train $d$ must

AQ: Please check Is anything missing in the equation a $\mathrm{J}+1$. be shifted backwards to provide the departure space for the delayed train as shown in Fig. 3. A MATLAB program is developed for timetabling algorithm as shown in Table 1 .

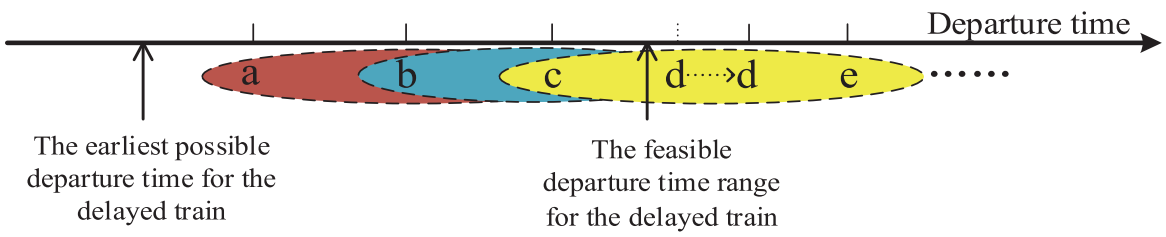

Fig. 3. Search strategy when $j=3$.

\subsection{Train platforming algorithm}

Carey and Crawford [7] and Lusby et al. [14] pointed out that the train platforming problem is a NP-hard problem and no universally valid algorithm can be found. It is necessary to develop an algorithm so that platforming plan due to change train timetabling can be taken into account in the decision process in the case of the 
Table 1. Train timetabling algorithm.

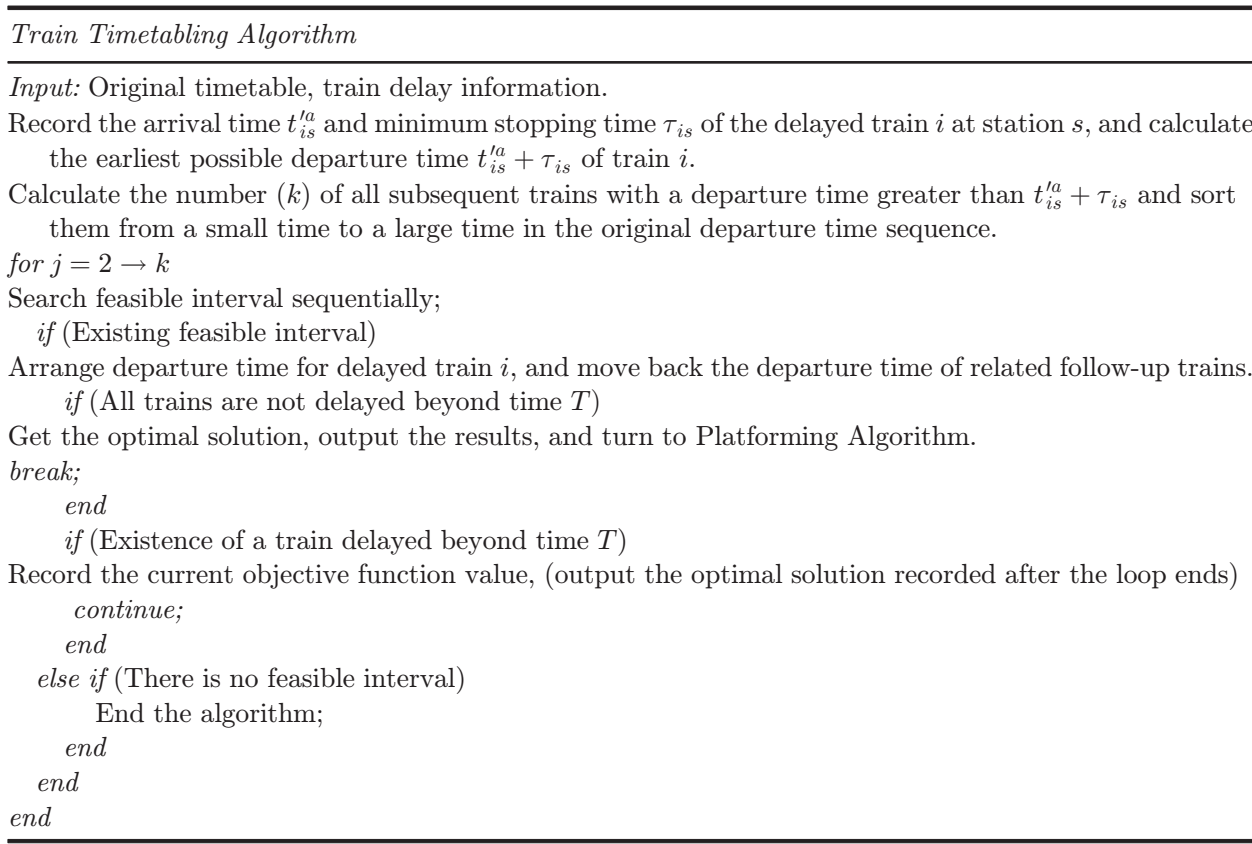

train delay. Currently, simulated annealing algorithm (SAA) [15] is widely used to solve such problems in searching for an optimal solution from a large number of possible alters. The analysis process of SAA starts from an initial high temperature transfers to a final low temperature based on cooling schedule, e.g. cooling rate, iteration number and temperature threshold. In this paper, the SAA is used to find platforming solution based on the new train schedule calculated by timetabling algorithm as described in Sec. 3.1. The general framework of the SAA is not presented in this paper. Details of SAA can be found in [15]. However, the solution of the SAA, the generation of the relative solution and the Metropolis criterion are discussed.

(1) The solution: according to the number of trains $(k)$ and the number of the platform tracks that can be used by the current station, a matrix $A_{k \times 2}$ is used to represent the solution of the algorithm, i.e.

$$
A_{k \times 2}=\left[\begin{array}{cc}
\text { train1 } & \text { track number } \\
\vdots & \vdots \\
\text { train } i & \text { track number } \\
\vdots & \vdots \\
\text { train } k & \text { track number }
\end{array}\right] \text {, }
$$


where the first column of the matrix $A_{k \times 2}$ represents the train number and the second column represents the platform track number occupied by the trains.

(2) Relative solution: change of the track numbers in the second column of matrix $A_{k \times 2}$ will produce a new matrix $A_{k \times 2}$ that constitutes the current solution.

(3) Metropolis criterion: the Metropolis criterion in simulation annealing algorithm is the acceptance criterion that is most commonly used to judge whether or not the newly generated domain solution is acceptable as the current solution. Suppose $P_{t}\left(A_{k \times 2} \Rightarrow A_{k \times 2}^{\prime}\right)$ as the probability of the new accepted solution at temperature $t$, then:

$$
\begin{aligned}
P_{t}\left(A_{k \times 2} \Rightarrow A_{k \times 2}^{\prime}\right) & = \\
& = \begin{cases}1, & \text { if } Z_{2}\left(A_{k \times 2}^{\prime}\right) \leq Z_{2}\left(A_{k \times 2}\right) \\
\exp \left(\frac{Z_{2}\left(A_{k \times 2}\right)-Z_{2}\left(A_{k \times 2}^{\prime}\right)}{t}\right), & \text { otherwise }\end{cases}
\end{aligned}
$$

A MATLAB program is also developed for platforming algorithm to find the solution as shown in Table 2 in which SAA [15] has been employed in the determination of platforming planning. In this case, initial temperature $=$ the number of trains, iteration number $=$ track number, cooling rate $=$ suitable track rate at the station, and temperature threshold = impact due to the train delay.

Table 2. Train platforming algorithm.

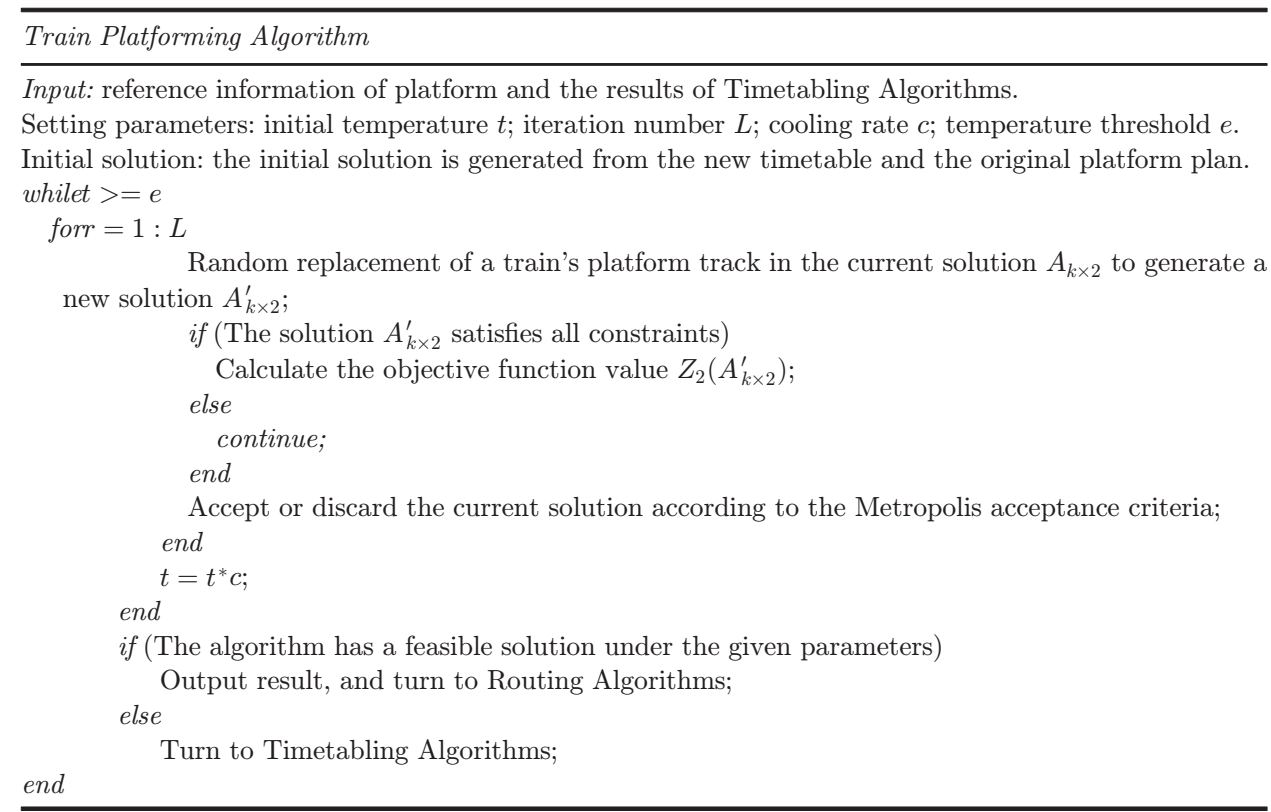




\subsection{Routing algorithm}

Once the platform tracks to be occupied by trains are determined, the routes connecting the platform tracks can be enumerated one by one according to the station structure. The process of train routing algorithm of a MATLAB program is shown in Table 3 .

Table 3. Train routing algorithm.

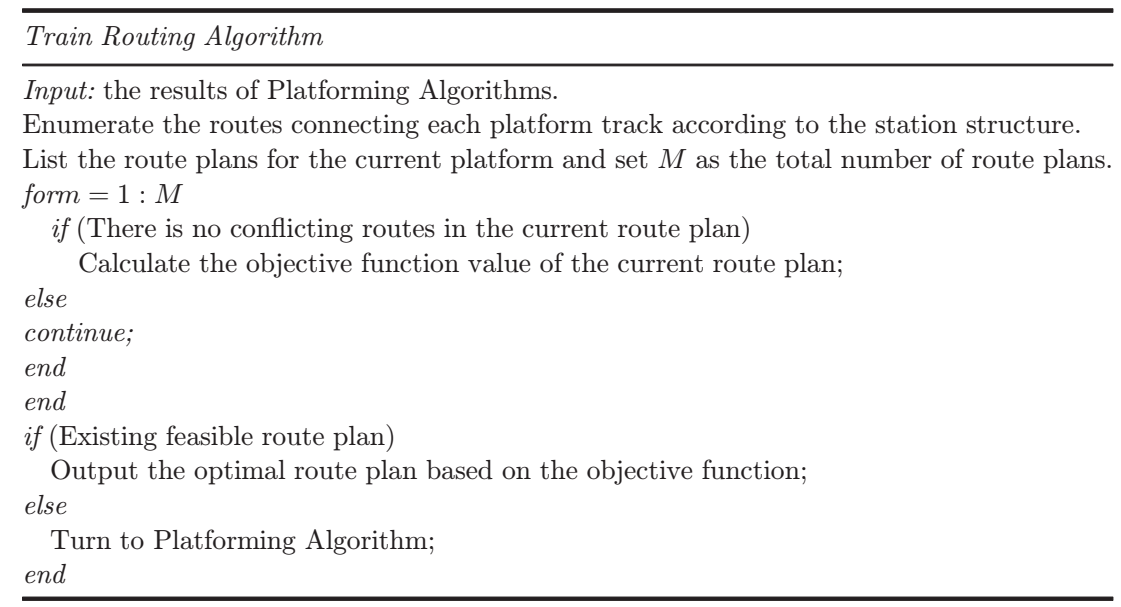

\subsection{Cooperative adjustment algorithm}

As described in Secs. 3.1-3.3, three sub-algorithms are used to form cooperative adjustment algorithm in order to coordinate adjustment of the delayed trains. For example, if a train is delayed, it would affect the timetabling, platforming and routing plans in each station in railway network because of such a delay. If there is a secondary delay in the adjustment process, the same adjustment strategy will be applied again for all of the trains in the railway network until a solution can be reached. By using the proposed cooperative adjustment algorithms quickly adjusting the train operation scheme and restoring the normal train order can be achieved. Figure 4 shows flow chart of the proposed cooperative adjustment algorithm. The process can be summarized as:

Step 1. input data and information of the delayed train $i$ at station $s$, and calculate the earliest possible departure time of train $i$.

Step 2. If the earliest possible departure time exists, move to Step 3. If the earliest possible departure time does not exist, move to Step 4.

Step 3. calculate platforming algorithm to search for a suitable platforming plan. If a suitable platforming plan exists, move to Step 5. Otherwise, move to Step 4. 


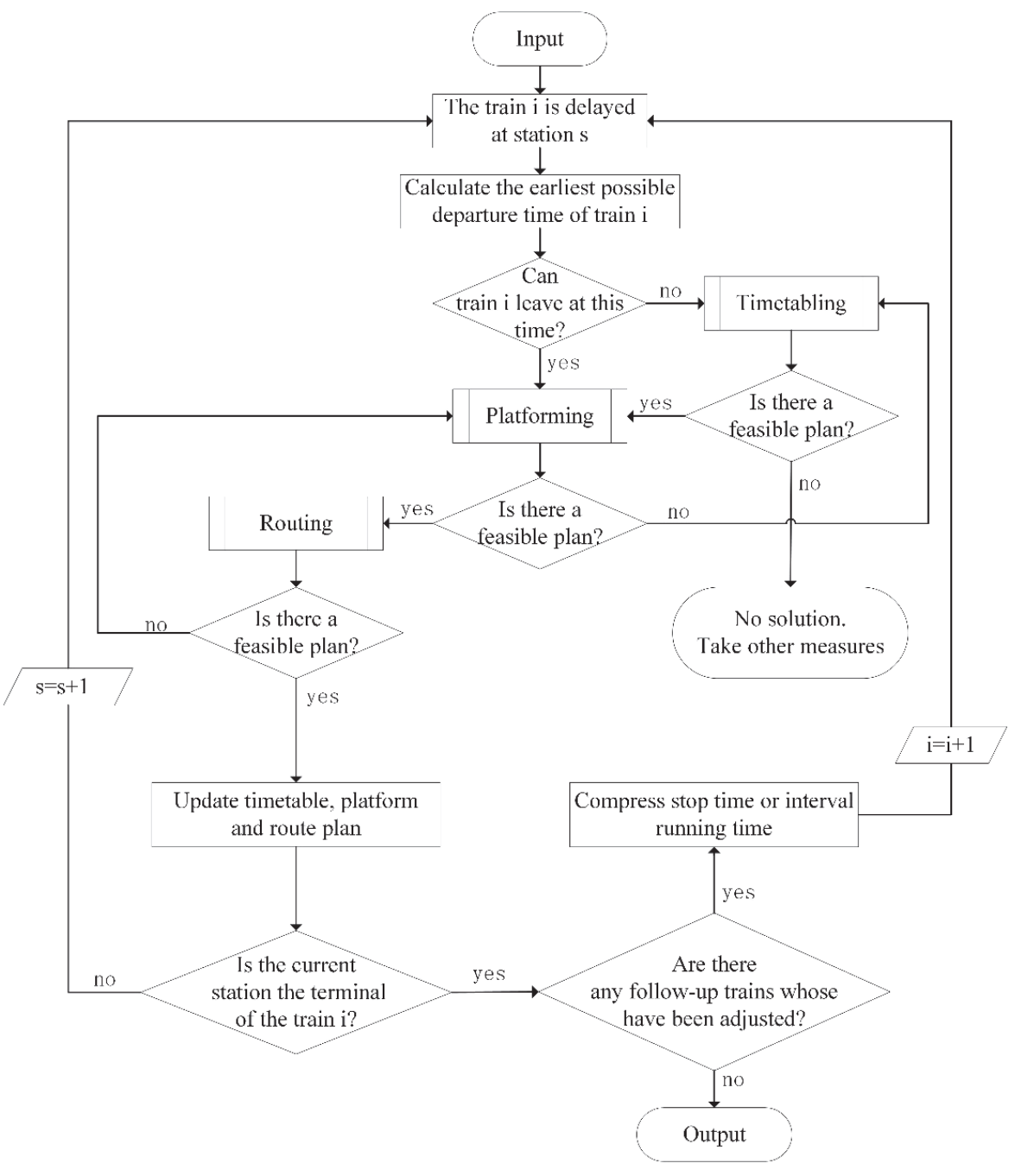

Fig. 4. Flow chart of cooperative adjustment algorithm.

Step 4. Search for a feasible timetabling plan. If it exists, move to Step 3 to calculate platforming algorithm. Otherwise, no solution can be found and end the process.

Step 5. Calculate routing algorithm to search for an available routing plan. If an available routing plan exists, move to Step 6. Otherwise, move to Step 3.

Step 6. If station $s$ is the terminal station of train $i$, move to Step 7. Otherwise, let $s=s+1$, move to Step 1 .

Step 7. Search for the affected trains by the delayed train $i$. Let $i=i+1$ and move to Step 1. Otherwise, end the process and output results. 


\section{Case Study}

A case study is presented in this section to demonstrate the application of the proposed method as described in Sec. 3. About 21 pairs of trains from five adjacent stations A, B, C, D and E between 12:00 and 16:00 are selected. Figure 5 shows the original train operation scheme.

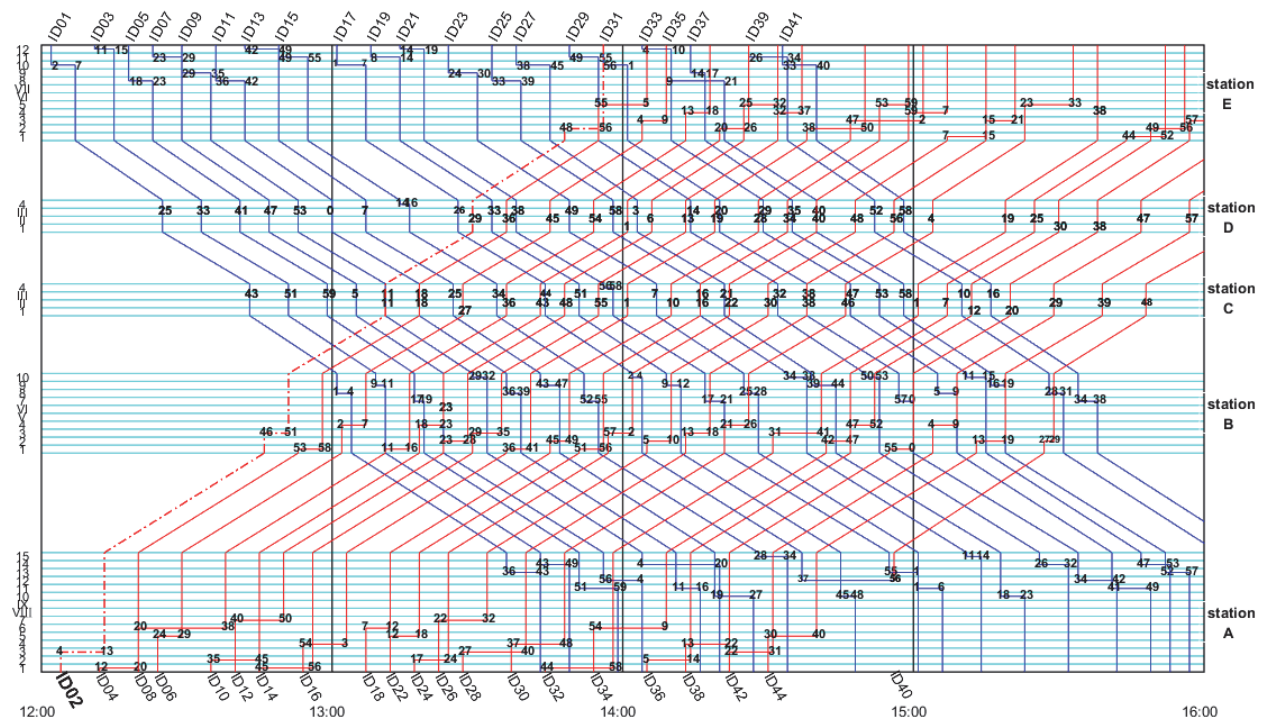

Fig. 5. Original train operation scheme.

As can be seen in Fig. 5, the number of platform tracks used at stations A, B, C, D and $\mathrm{E}$ are 15, 10, 4, 4, and 12, respectively, with the main line as the segmentation. The upward and downward trains stop in the corresponding receiving/departure yard. The tracking interval in all these five stations in the upward directions are both 5 min, the arrival interval of stations $\mathrm{A}, \mathrm{B}$ and $\mathrm{E}$ is $5 \mathrm{~min}$, the continuous non-stop passing interval of stations $\mathrm{C}$ and $\mathrm{D}$ is $3 \mathrm{~min}$. The interval between two trains in these five stations using a same platform track is $8 \mathrm{~min}$.

Due to an unexpected event, the upward train ID02 was delayed for $28 \mathrm{~min}$, and the arrival time of station A was delayed from 12:04 pm to 12:32 pm. On the premise of meeting the above requirements of safe interval and technical operation time of each station, the adjustment scheme obtained by using the proposed method is shown in Fig. 6. In this case, when $T=60$ mins, weight factor $\alpha=1000$ in Eq. (3), and other two optimization weight factors $\beta_{1}=0.9999$ and $\beta_{2}=1-\beta_{1}=0.0001$ in Eq. (13).

As can be seen from Fig. 6, in the section between stations A and E, train ID16 is affected by train ID02 because of its delay, and train ID16 is restored to the original 


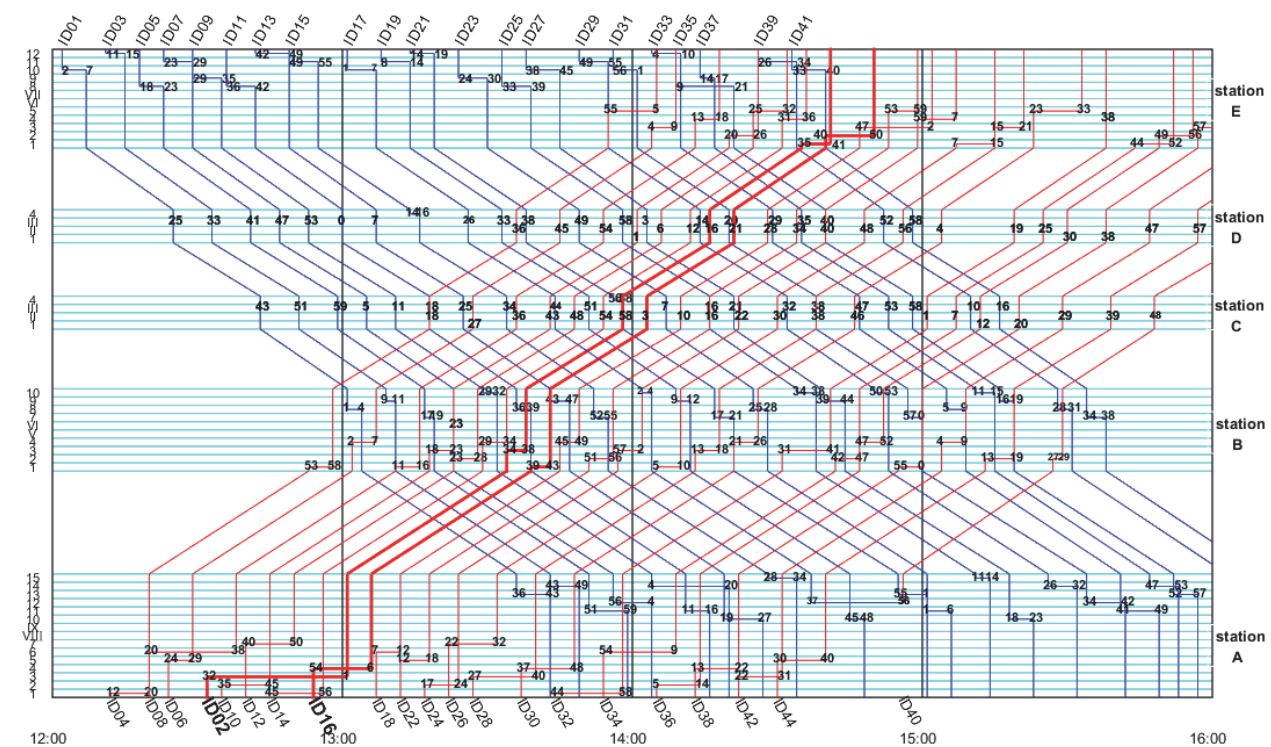

Fig. 6. Adjusted train operation scheme.

planned departure time at station E. The total number of delayed trains is 5 , i.e. 2 trains are delayed in station A, 2 trains in station $\mathrm{B}$ and 1 train in station $\mathrm{E}$, and the total delay time is $10 \mathrm{~min}$.

The platform plan in stations $\mathrm{A}$ and $\mathrm{E}$ has little change in the process of adjustment, while the platform plan in stations $\mathrm{C}$ and $\mathrm{D}$ has not changed.

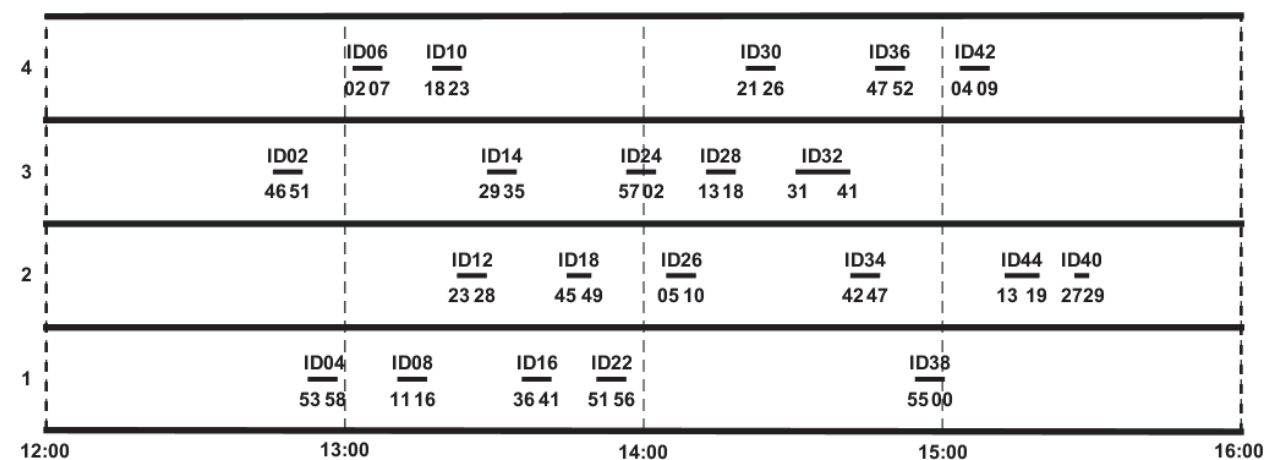

Fig. 7. Original platform plan in the upward direction of station B.

Figures 7 and 8 show the original platform plan and the adjusted platform plan in the upward direction of station B. 


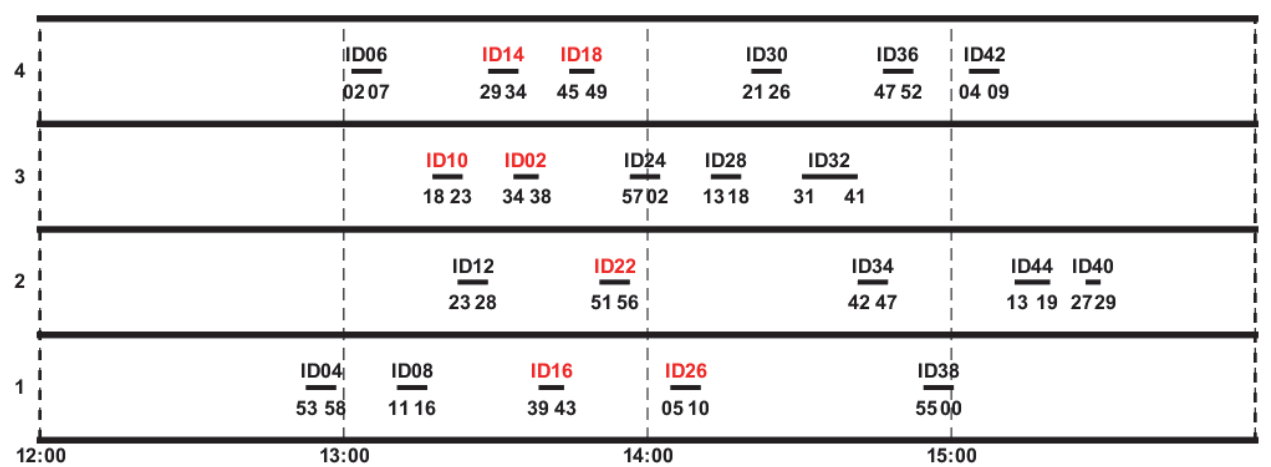

Fig. 8. Adjusted platform plan in the upward direction of station B.

As can be seen from Figs. 7 and 8, platform plan for trains ID14, ID18, ID10, ID16, ID22 and ID26 have been changed due to the delay of train ID02. Compared with the original platform plan, the volatility and the equalization of the platform plan can be guaranteed, which can effectively reduce the adjustment workload caused by the delay of train ID02. It should be noted that the case study only shows the process of adjustment because of the delay caused by a train. If the delay is caused by multiple trains, adjustments should be undertaken separately in the same way. For example, if the delay is caused by two trains, adjustment should be undertaken to analyze the first train delay to find a solution, and then to use such a solution as the initial condition to examine the second train delay, and finally the final solution can be found. In other words, If the delay is caused by multiple trains, the adjustments can be done to analyze each train delay one by one separately following the way as demonstrated in case study to find the final solution.

\section{Conclusions}

This paper presents a proposed cooperative adjustment algorithm that can be used in the case of the train delay to adjust the train operation plan, restore the train operation, and reduce the impact of train delay effectively in the railway network. MATLAB programs have been developed in order to calculate solutions based on three sub-algorithms of timetabling, platforming and routing. The results from case study indicate that the proposed cooperative adjustment algorithm can realize the coordinated adjustment of train timetabling, platforming and routing plans quickly and efficiently. Compared with the traditional methods of hierarchical adjustment through multiple single models, the total number of delayed trains and the total delay time of trains can be well controlled by using the proposed method. Railway operational monitor and control system is currently developed based on sets of rules and regulations made by the national authorities and classification societies, e.g. Health \& Safety Executive Department, Rail Safety and Standard Board for 
17

AQ: Please provide closing quotes.

Transportation, Railway Operation Co. etc. However, in many cases, the current railway monitor and control system cannot be applied to analyze train delay accidents and incidents effectively and efficiently because the railway always operates in a very changeable environment which involves human interaction combined with human judgement, experience and engineering knowledge. The proposed methodology can formulate and transform domain human judgement, experience and engineering knowledge to be the knowledge base as adjustment standard and criteria, which can enhance railway monitor and control system to be applied more intelligently. Therefore, the proposed methodology can also be incorporated into the intelligent railway monitor and control system so that train operation management can be improved while increasing railway safety.

\section{Acknowledgments}

This work describes herein is part of research projects funded by the National Natural Science Foundation of China (Grant No. 71971220), the Natural Science Foundation of Hunan Province, China (Grant No. 2019JJ50829), and Highways England on "New Methodology for Maintenance Decision Making of Structures of Transport Infrastructures (Grant No. 546037-PMRB13) with technical support from major industry players. Their support is gratefully acknowledged.

21

22

23

24

25

26

\section{References}

1. S. Dindar, S. Kaewunruen and M. An, Rail accident analysis using large-scale investigations of train derailments on switches and crossings: Comparing the performances of a novel stochastic mathematical prediction and various assumptions, Eng. Fail. Anal. 103 (2019) 203-216.

2. F. Corman, A. D'Ariano, A. D. Marra, D. Pacciarelli and M. Samá, Integrating train scheduling and delay management in real-time railway traffic control, Transport. Res. E, Logist. Transp. Rev. 105 (2017) 213-239.

3. X. Luan, Y. Wang, B. De Schutter, L. Meng, G. Lodewijks and F. Corman, Integration of real-time traffic management and train control for rail networks-Part 1: Optimization problems and solution approaches, Transport. Res. B, Methodol. 115 (2018) 41-71.

4. V. Cacchiani, D. Huisman, M. Kidd, L. Kroon, P. Toth, L. Veelenturf and J. Wagenaar, An overview of recovery models and algorithms for real-time railway rescheduling, Transport. Res. B, Methodol. 63 (2014) 15-37.

5. L. Yang, J. Qi, S. Li and Y. Gao, Collaborative optimization for train scheduling and train stop planning on high-speed railways, Omega 64 (2016) 57-76.

6. M. Carey and S. Carville, Scheduling and platforming trains at busy complex stations, Transport. B, Methodol. 37(3) (2003) 195-224.

7. M. Carey and I. Crawford, Scheduling trains on a network of busy complex stations, Transport. Res. B, Methodol. 41(2) (2007) 159-178.

8. Y. Zhang, D. Lei and M. Liu, Scheduling model and algorithm for track application in railway station, J. China Railw. Sci. 31(2) (2010) 96-100 (in Chinese).

9. M. Samà, C. Meloni, A. D'Ariano and F. Corman, A multi-criteria decision support methodology for real-time train scheduling, Rail Transp. Plan. Manage. 5(3) (2015) 146-162. 
10. J. Törnquist and J. A. Persson, N-tracked railway traffic re-scheduling during disturbances, Transport. Res. B, Methodol. 41(3) (2007) 342-362.

11. Q. F. Zeng, Y. G. Zhang and D. Y. Lei, An experimental analysis on dispatching rules for the train platforming problem in busy complex passenger stations, Prome -Traffic Transport. 29(4) (2017) 411-423.

12. D. Y. Lei, Y. G. Zhang and X. Y. Wang, Coupled due windows scheduling model and algorithm of carriage operations arrangements in railway technology stations, China Railw. Soc. 32(4) (2010) 1-7 (in Chinese).

13. M. Si, J. K. Sun and G. Y. Lu, Optimization and adjustment of train route allocation scheme for high speed railway stations, China Railw. Sci. 39(1) (2018) 122-130 (in Chinese).

14. R. Lusby, J. Larsen, D. Ryan and M. Ehrgott, Routing trains through railway junctions: A new set-packing approach, Transport. Sci. 45(2) (2011) 228-245.

15. S. Kirkpatrick, Optimization by simulated annealing: Quantitative studies, Stat. Phys. 34(5-6) (1984) 975-986. 